\title{
A time-driven transmission method for well logging networks
}

\author{
Wu Ruiqing ${ }^{1 *}$, Chen Wei ${ }^{1}$, Chen Tianqi ${ }^{1}$ and Li Qun ${ }^{2}$ \\ ${ }^{1}$ School of Electronic Engineering, University of Electronic Science and Technology of China, Sichuan 610054, China \\ ${ }^{2}$ Research \& Technical Center, China Oilfield Services Limited, P.O. 232 Beijing 101149, China
}

\begin{abstract}
Long delays and poor real-time transmission are disadvantageous to well logging networks consisting of multiple subnets. In this paper, we proposed a time-driven transmission method (TDTM) to improve the efficiency and precision of logging networks. Using TDTM, we obtained well logging curves by fusing the depth acquired on the surface, and the data acquired in downhole instruments based on the synchronization timestamp. For the TDTM, the precision of time synchronization and the data fusion algorithm were two main factors influencing system errors. A piecewise fractal interpolation was proposed to fast fuse data in each interval of the logging curves. Intervals with similar characteristics in curves were extracted based on the change in the histogram of the interval. The TDTM is evaluated with a sonic curve, as an example. Experimental results showed that the fused data had little error, and the TDTM was effective and suitable for the logging networks.
\end{abstract}

Key words: Data fusion, fractal interpolation, histogram, packet switch, timestamp

\section{Introduction}

Due to good performance in device interconnection, transmission efficiency and universal application compared with those of other field buses, computer networks play an increasingly important role in well logging systems, and become one of the most important characteristics of the developing fifth generation network well logging systems (Xiao et al, 2003; Tang, 2007; Wang et al, 2006). Although the switched Ethernet is a perfect solution for providing high throughput, short delay and low delay jitter to meet the demands of multi-measurement and control networks (Lian, 2001), the well logging network is a typical remote measurement and control network consisting of switched Ethernet and routers (Wu et al, 2005), due to strict environment limitations, such as wiring connection, transmission distance, and device dimension. One simple instance is that the surface equipment composes a control subnet, the surface telemetry instruments (or surface gateway) and the downhole wireline telemetry cartridge (or downhole gateway) compose the transmission subnet, and all the downhole instruments compose an instrument subnet. We discovered that the logging network is a network focusing on measurement, which needs to acquire accurate data and record the corresponding measurement parameters, such as time, depth, and length. The parameter data are transmitted from the surface equipment to downhole instruments through multi-hop networks. This process degrades the network utilization and increases delay, therefore it is impossible to meet the real-time demands. Some methods to increase line

*Corresponding author. email: reeching@163.com

Received December 7, 2008 speed, reduce time delay and optimize the router software cannot improve the real-time performance effectively, and are also very difficult to implement.

However, little research on this problem has been published. In this paper, we presented a time-driven transmission method (TDTM) as an effective solution based on accurate time synchronization and fast data fusion. During data acquisition, the real-time transmission of parameter information from the surface equipment to downhole instruments was not required. The TDTM is used to reduce network traffic and improve network efficiency for high precision measurements.

\section{Time-driven transmission model}

\subsection{Data flow chart}

Due to long delays and low transmission rates, the existing logging systems mainly work in a "request-reply" mode, as illustrated in Fig. 1(a) (Meng et al, 2003). The surface equipment sends commands and the depth parameter as a "request" to downhole instruments, and then the instruments start to acquire data at the depth and transmit the data as a "reply" to the surface equipment after a delay. Finally, the surface equipment obtains the logging curve, which describes the change of measured data with depth. After transmitting commands to downhole instruments, the surface equipment is inactive until receiving the acquired data or state information from downhole instruments. With the increase of downhole sensors and parallel processing demand, the "request-reply" mode becomes inefficient in logging applications.

The well logging network using the TDTM consists of the surface control center (SCC), the surface time-depth 
acquiring unit (TDAU), the surface time-depth-data fusion unit (TDDFU), system time synchronization unit (STSU), transmission network (TN), and downhole logging tools (DLT). The SCC forms the work command tables and sends them to downhole instruments by networks. The STSU accurately synchronizes each node's clock of downhole instruments and surface equipment. According to the predetermined depth interval, when hardware interrupts of sampling depth occur, the TDAU acquires the depth and corresponding timestamp, and then obtains a time-depth pair $\left(t_{i}, d_{i}\right)$. Many time-depth pairs compose the time-depth sequence $\langle t, d\rangle$. We assume that the time-depth sequence $<t, d>$ satisfies a function $d=f(t)$. Generally, $d=f(t)$ is a piecewise linear function. Even if the intervals of depth interrupt are equal, when the speed of the logging cable changes, the intervals of timestamps are not always equal.

The downhole instruments record the measured data $R_{i}$ corresponding to the time $T_{i}$ and obtain the timedata pair $\left(T_{i}, R_{i}\right)$. Many time-data pairs compose a timedata sequence $\langle T, R>$. Then, the telemetry instruments transmit the sequence $\langle T, R>$ to the TDDFU. We assume that the time-data sequence $\langle T, R>$ satisfies a function $R=g(T)$. Because of the difference in working mechanism of telemetry instruments, there are much data acquired at unequal time intervals. The TDDFU fast fuses the $\langle t, d\rangle$ and $\langle T, R>$ sequences, and finally obtains the depth-data sequence $\langle d, r\rangle$, i.e., the logging curve. Fig. 1(b) shows the basic flowchart of the TDTM.

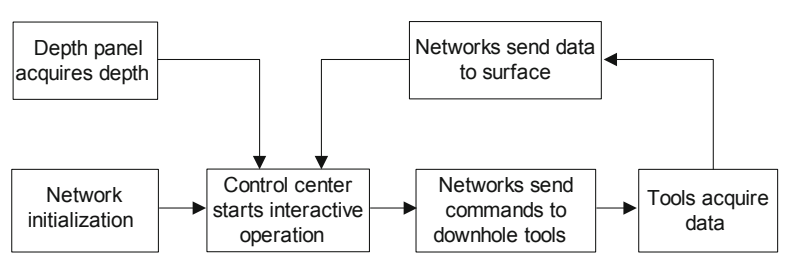

(a) Basic flowchart of the traditional model

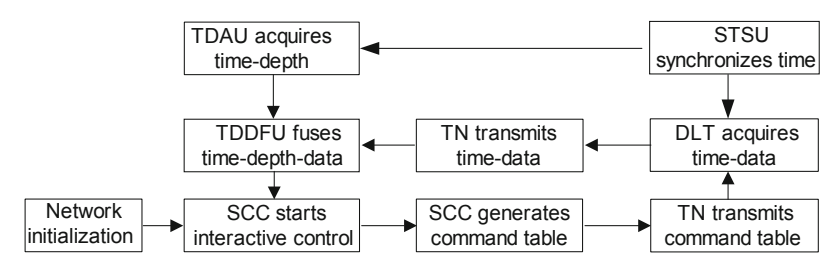

(b) Basic flowchart of the TDTM model

Fig. 1 Well logging data flowchart

Compared with the "request-reply" mode, the TDTM uses the data fusion method based on the time synchronization unit and data fusion unit. The TDDFU is an important module in TDTM for fast and accurate data processing. In the following, we present a fast data fusion algorithm based on the analysis of the mapping model of time-depth-data.

\subsection{Time-depth-data mapping}

After the time is synchronized in the logging network, we can obtain the fused depth-data sequence by the following equation:

$$
\left\{\begin{array}{l}
d=f(t) \\
R=g(T) \\
t=T \\
r=R
\end{array}\right.
$$

The logging curve is $r=g\left(f^{-1}(d)\right)=h(d)$, but actually it is very difficult or impractical to obtain $r=h(d)$. In order to explain more clearly, we analyze the mapping relationship, assuming that the measured data $r$ is one dimensional, as shown in Fig. 2(a).

In Fig. 2, $\left(t_{i}, d_{i}\right)$ and $\left(t_{j}, d_{j}\right)$ are from the time-depth sequence, $\left(T_{i}, R_{i}\right)$ and $\left(T_{j}, R_{j}\right)$ are from the time-data sequence, and $\left(d_{i}, r_{i}\right)$ and $\left(d_{j}, r_{j}\right)$ are to be obtained. Because the timedata sampling and the time-depth sampling are asynchronous, the sampling intervals of the two sequences are unequal. Furthermore, the forms of $g(T)$ and $f(t)$ are unknown, so $h(d)$ has no explicit expression. Because $T_{i}$ and $t_{i}$ are different, the goal of the depth-data fusion is to obtain the optimal $(t, r)$ from $(T, R)$ by an effective interpolation, as shown in Fig. 2(b).

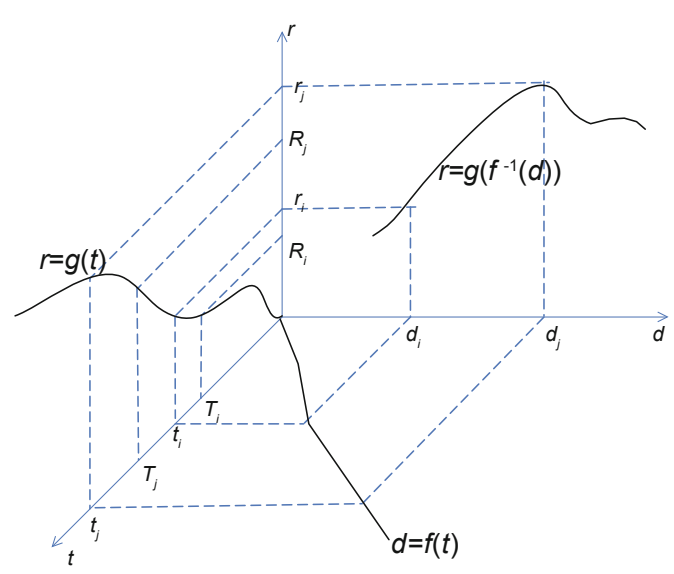

(a) Mapping of two sequences

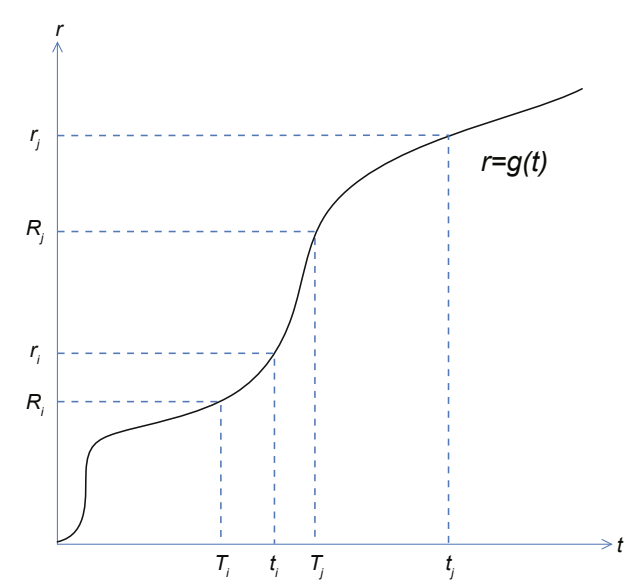

(b) Time-data interpolation

Fig. 2 Time-depth-data mapping 
Usually, $d=f(t)$ is piecewise linear, and $r=h(d)$ and $r=g(t)$ have similar monotonicity, although they are in different variable domains. This conclusion can be proven simply as follows: Because $d=f(t)$ is piecewise linear, we assume that it has the form $d=f(t)=a t+b$ in its linear interval, where $a$ is a positive constant (if $a$ is negative, the conclusion will be the same), and $b$ is an undetermined constant. Then, $t=d / a-b / a$ and $r=g(d / a-b / a)=h(d)$, so $r=h(d)$ and $r=g(t)$ have a similar monotonicity. This conclusion is very important to the selection of the interpolation algorithm in the time domain to obtain $\left(t_{i}, r_{i}\right)$ from $\left(T_{i}, R_{i}\right)$.

\section{TDTM error analysis}

We focus on the main factors influencing the performance of the TDTM. In fact, the data processing shows that $t$ and $T$ are not always equal, and do not satisfy Eq. (1). We can describe their relation by the following equation:

$$
t=T+\Delta t+\Delta s
$$

where $\Delta t$ is the error resulting from synchronization, and $\Delta s$ is the error from the asynchronous data acquisition of the surface equipment and downhole instruments. Thereby, there are two factors influencing the performance of the TDTM. One factor is the precision of the network time synchronization, and the other is the data fusion algorithm. Much work has been done on the methods to highly synchronize the network time. In this paper, an efficient interpolation algorithm was proposed to minimize the error from asynchronous acquisition. The discussion in the following section will focus on the influence of $\Delta t$ and $\Delta s$.

\subsection{Time synchronization error}

We suppose that there is an absolute accurate time $t$, and the time synchronization error between surface equipment and downhole instruments is $\Delta t$. When $\left(t_{i}, d_{i}\right)$ is acquired on the surface at $t_{i}$, but $\left(t_{i}+\Delta t, r_{i}\right)$ is recorded in the downhole, so they will be mistaken as the measurement data at different depth. If $\Delta t$ increases, it will result in an increase of depth error. In the TDTM, $t_{i}$ is stored in a timestamp field of the packet. There are two basic attributes related to the timestamp. One is time granularity, which indicates the resolution of the system timer. In our experiment, the granularity of the platform named ELIS-800 is $1.6 \mu \mathrm{s}$, which means that the time counter increases by one per $1.6 \mu \mathrm{s}$. The other attribute is the bit number allocated for storage, and the bit number of ELIS-800 is set to 32 bit.

Generally, the cable speed, defined by $v$, is a piecewise constant less than $30 \mathrm{~m} / \mathrm{min}$. The depth $d$ and time $t$ satisfy the following equation:

$$
d(t)=\int_{0}^{t} v \mathrm{~d} t+d_{0}
$$

Therefore, the depth error is $\Delta d=v \Delta t$. When the time error $\Delta t$ maintains a specific value, if $v$ increases, $\Delta d$ will become greater. For example, in PEXWL (Platform Express Integrated Wireline Logging), the cable travels at a speed of $v=3600 \mathrm{ft} / \mathrm{hr}=0.3048 \mathrm{~mm} / \mathrm{ms}$. In the ELIS-800 experimental platform, the maximum $\Delta t$ is equal to $10 \mathrm{~ms}$, so the maximum depth error achieves $\Delta d=3.034 \mathrm{~mm}$, which is acceptable in the logging system. Furthermore, the time delay in logging network is more than $100 \mathrm{~ms}$, which results in a more serious depth error without the TDTM.

\subsection{Influence of interpolation algorithm on error}

There are many interpolation algorithms providing different complexities and levels of performance, such as bilinear, cubic spline, Lagrange, and Hermite interpolation. The optimum interpolation algorithm should retain the characteristics of the sequence $\left(T_{j}, R_{j}\right)$ to the greatest extent (Burnside and Parks, 1990). The data acquired by telemetric instruments, such as sonic waves, gamma rays, and natural electric potentials, reflect the attributes of geological objects. The existing literature and the actual analysis results show that different logging curves have different characteristics, and most logging curves have obvious fractal characteristics in the entire depth domain (Li and Xiao, 2002; Li, 2005; Lu and $\mathrm{Li}, 1996)$, so fractal interpolation can be used to improve the resolution.

Usually, fractal dimension is a measure to determine whether the logging curve has fractal characteristics. Moreover, the fractal characteristics of logging curves change with geological structure. Well logging curves have different fractal characteristics in different depth intervals, even in the same well (Hewett, 1986; Liu et al, 2004). Table 1 gives the statistics of fractal dimensions of different interval lengths from a sonic sequence as shown in Fig. 4(a). This sonic sequence is recorded from the depth $2079 \mathrm{~m}$ to $2487 \mathrm{~m}$, including 2675 points. The fractal dimensions are calculated by a box dimension algorithm.

Table 1 Fractal dimensions of different interval lengths

\begin{tabular}{cccc}
\hline Interval length & Max. & Min. & Average \\
\hline 50 & 1.1551 & 1.0577 & 1.1192 \\
100 & 1.2042 & 1.1373 & 1.1694 \\
150 & 1.2357 & 1.1743 & 1.2055 \\
200 & 1.2557 & 1.1977 & 1.2309 \\
2675 & 1.52 & 1.52 & 1.52 \\
\hline
\end{tabular}

From Table 1, we can see that the fractal dimension is not a constant and shows the different fractal features in different intervals. Therefore, piecewise fractal interpolation should be used to retain local characteristics and improve accuracy of data fusion.

\section{Fast data fusion}

Existing literature has focused on calculating the fractal dimension, discussing the performance of interpolation function, and deriving the error of fractal interpolation 
function ( $\mathrm{Li}$ and Xiao, 2002; Li, 2005; Sha and Liu, 2004). Directly calculating the fractal dimension in real-time analysis of the logging curves has some disadvantages, such as large iterations, poor real-time performance and high computation load in the interpolation interval. We present a fast data fusion algorithm based on the change in the histogram of timedata. This algorithm has more advantages, such as retaining local characteristics, reducing the usage of resources, and improving computation speed.

The histogram of data with the same fractal characteristics usually has a dense, symmetrical or quasi-symmetrical distribution, such as normal distribution or box-type ( $\mathrm{Li}$ and Xiao, 2002). Therefore, we propose a method to extract the intervals for piecewise interpolation based on the change of histogram in the distribution features. In each extracted interval, the self-affine fractal interpolation method is used to obtain a high-resolution curve.

\subsection{Logging curve piecewise extraction}

\subsubsection{Extract statistical parameter}

Firstly, we obtained the histogram of $M_{n}$ logging data $R_{i}$ in the interval $\varphi_{n}$, and then calculated the mean $\mu_{n}$ and variance $\sigma_{n}$ of $R_{i}$. Then, we extracted the highest peak $P_{m}$ in the histogram and its corresponding measurement value $y_{m}$. Finally, we calculated the peak drift $y_{m}-y_{m-1}$ compared with the previous interpolation interval $\varphi_{n-1}$.

\subsubsection{Determination of PP-skewness and kurtosis}

The PP-skewness and kurtosis are two properties of the stochastic variable distribution. The PP-skewness defined by $k$ is a measure of symmetry distribution (Chen and Cui, 2004). The PP-kurtosis defined by $g$ is a measure of the density of the probability distribution. The $k$ of normal distribution is usually 0 , and $g$ is 3 . If $k$ is far away from 0 or $g$ far away from 3 , we can conclude that it may be much different from the normal distribution. The PP-skewness and kurtosis of stochastic logging data $R_{1}, R_{2}, \cdots, R_{m}$ in $\varphi_{n}$ are calculated according to the following equation:

$$
\left\{\begin{array}{l}
k_{n}=\sum_{i=1}^{m}\left(R_{i}-\bar{R}\right)^{3} /\left(\sum_{i=1}^{m}\left(R_{i}-\bar{R}\right)^{2}\right)^{\frac{3}{2}} \\
g_{n}=\sum_{i=1}^{m}\left(R_{i}-\bar{R}\right)^{4} /\left(\sum_{i=1}^{m}\left(R_{i}-\bar{R}\right)^{2}\right)^{2}
\end{array}\right.
$$

where $\bar{R}=\frac{1}{m} \sum_{i=1}^{m} R_{i}$. If a large change exists from $k_{n-1}<0$ to $k_{n}>0$, or from $k_{n-1}>0$ to $k_{n}<0$, which is a jump round the zero point, a new fractal interpolation should start in the interval $\varphi_{n}$

\subsubsection{Determination of peak and variance accumulative drift}

The peak accumulative drift from interval $\varphi_{l}$ to $\varphi_{n}$ is defined by $P(l, n)=\sum_{i=l}^{n}\left(y_{i}-y_{i-1}\right)$, where $n>l$, and variance accumulative drift from interval $\varphi_{l}$ to $\varphi_{n}$ is defined by $F(l, n)=\sum_{i=l}^{n}\left(\sigma_{i}-\sigma_{i-1}\right)$, where $n>l$. If any one condition in the following equations is satisfied:

$$
\left\{\begin{array}{l}
\left|P(l, n)-\left(P_{\max }-P_{\min }\right) / 2\right|>\alpha \cdot\left(P_{\max }-P_{\min }\right) / 2 \\
\left|P(l, n)-\left(P_{\max }-P_{\min }\right) / 2\right|<\left(P_{\max }-P_{\min }\right) /(2 \alpha) \\
\left|F(l, n)-\left(F_{\max }-F_{\min }\right) / 2\right|>\beta \cdot\left(F_{\max }-F_{\min }\right) / 2 \\
\left|F(l, n)-\left(F_{\max }-F_{\min }\right) / 2\right|<\left(F_{\max }-F_{\min }\right) /(2 \beta)
\end{array}\right.
$$

where, $P_{\min }=\min (P(l, l+1), \cdots, P(l, n-1))$,

$P_{\text {max }}=\max (P(l, l+1), \cdots, P(l, n-1))$,

$F_{\min }=\min (F(l, l+1), \cdots, F(l, n-1))$,

$F_{\max }=\max (F(l, l+1), \cdots, F(l, n-1))$, then $\varphi_{n}$ has a different distribution characteristic from $\varphi_{l}, \ldots, \varphi_{n-1}$, and a new piecewise fractal interpolation starts in $\varphi_{n}$. Peak and variance accumulative drift are used to detect whether current intervals have similar characteristics or have gradual changes compared with the previous interval.

\subsection{Piecewise fractal interpolation and fusion}

Supposing that there are $M_{n}$ points in the interpolation interval $\varphi_{n}$ and $N$ data points in the mapping interval, the general form of the one-dimensional self-affine interpolation function is as follows:

$$
\omega_{i}\left[\begin{array}{l}
x \\
y
\end{array}\right]=\left[\begin{array}{cc}
a_{i} & 0 \\
c_{i} & d_{i}
\end{array}\right]\left[\begin{array}{l}
x \\
y
\end{array}\right]+\left[\begin{array}{l}
e_{i} \\
f_{i}
\end{array}\right], \text { where }\left|d_{i}\right|<1
$$

The detailed procedure of iterative interpolation can refer to two papers (Manousopoulos et al, 2008; Mazel and Hayes, 1992). The piecewise fractal interpolation interval $\varphi_{n}$ slides continuously with receiving the real-time data. It is therefore the so-called sliding window piecewise fractal interpolation. A higher-resolution time-data curve $\left\langle T^{\prime}, R^{\prime}\right\rangle$ is obtained by piecewise fractal interpolation. However, the expected $t_{i}$ in Fig. 2 may not be at $T_{i}$ in the $\left\langle T^{\prime}, R^{\prime}\right\rangle$. To solve this problem, the neighboring linear interpolation is taken to obtain $\left(t_{i}, r_{i}\right)$ from the two nearest values, i.e., the previous $\left(T_{m}^{\prime}, R_{m}^{\prime}\right)$ and the next $\left(T_{n}^{\prime}, R_{n}^{\prime}\right)$. Therefore, the fusion curve $<\tilde{d}, \tilde{r}>$ is obtained from $\langle t, d\rangle$ and $\langle t, r\rangle$.

\section{Simulation and results}

In order to evaluate the time-depth-data fusion algorithm, a sonic curve acquired in the depth of 2079-2487 $\mathrm{m}$ is given as a reference sequence $\langle d, r>$, whose depth sampling interval is $0.1524 \mathrm{~m}$. The simulation of depth-data fusion adopts the model shown in Fig. 3. After obtaining the time-depth sequence $\langle t, d\rangle$ and time-data sequence $\langle T, R>$, the $\langle\tilde{d}, \tilde{r}\rangle$ sequence is gained by piecewise fractal interpolation and fusion, where the threshold parameters in Eq. (5) are set to $\alpha=1.25, \beta=2$. Finally, the error curve $<d, \Delta \tilde{r}>$ between the $<d, r>$ and $<\tilde{d}, \tilde{r}>$ sequences is calculated. 


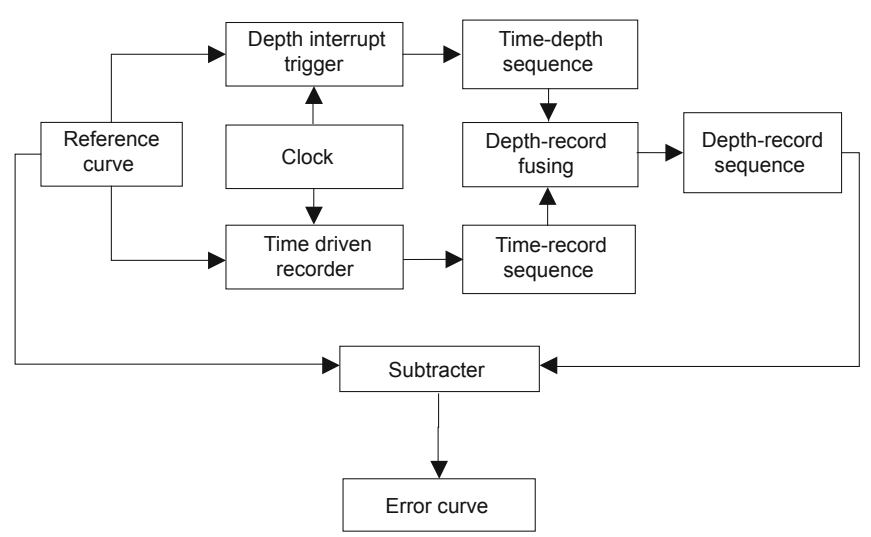

Fig. 3 Flowchart of time-depth-data simulation model

In order to compare the performance of piecewise fractal interpolation, we only use a linear interpolation method to obtain $\left(t_{i}, r_{i}\right)$ from the two nearest values, i.e., the previous $\left(T_{i}, R_{i}\right)$ and the next $\left(T_{j}, R_{j}\right)$ shown in Fig. 2, and then obtain the other fused curve $\langle d, \bar{r}\rangle$ and corresponding error curve $<d, \Delta \bar{r}\rangle$. The experimental results are illustrated from Fig. 4(b) to Fig. 4(i).

From the results shown in Fig. 4, the reference sonic curve shown in Fig. 4(a) has a large and continuous amplitude change at the depth of approximately $2200 \mathrm{~m}$. When the timedepth sequence is linear, the time-data curve has a similar monotonic property to the reference sequence as shown in Fig. 4(b) and Fig. 4(c). The variance and peak accumulative drift have a large jump at the depth of approximately $2200 \mathrm{~m}$, which indicates an evident change in distribution shown in Fig. 4(d) and Fig. 4(e). The PP-skewness and PP-kurtosis (both in a small amplitude) curves shown in Fig. 4(f) accord with the characteristics of dense distribution. After the piecewise fractal interpolation and fusion, the error between the fused curve shown in Fig. 4(g) and the reference curve is smaller as a whole, compared with simple linear interpolation, as illustrated in Fig. 4(h) and Fig. 4(i).

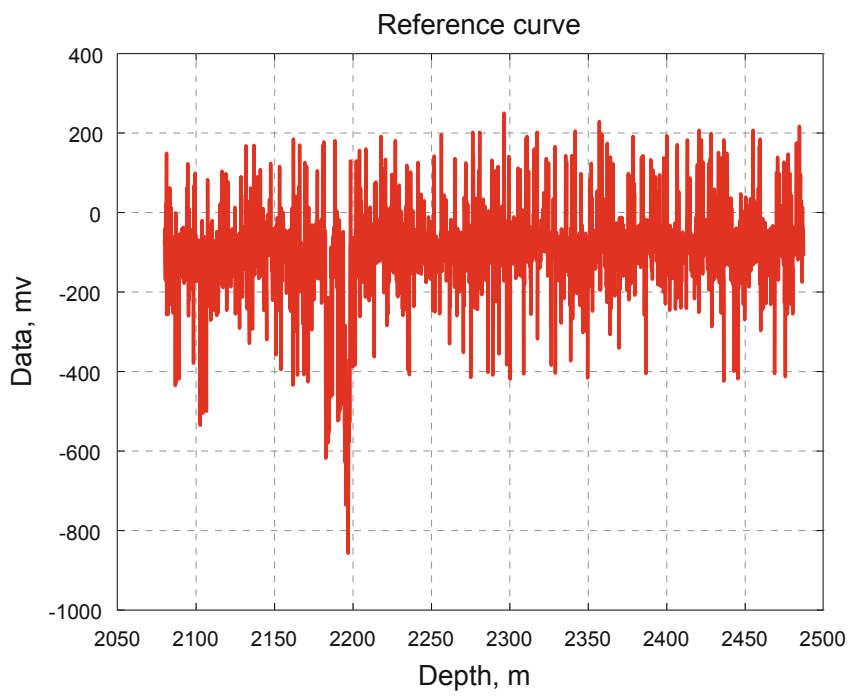

(a) Reference sonic curve

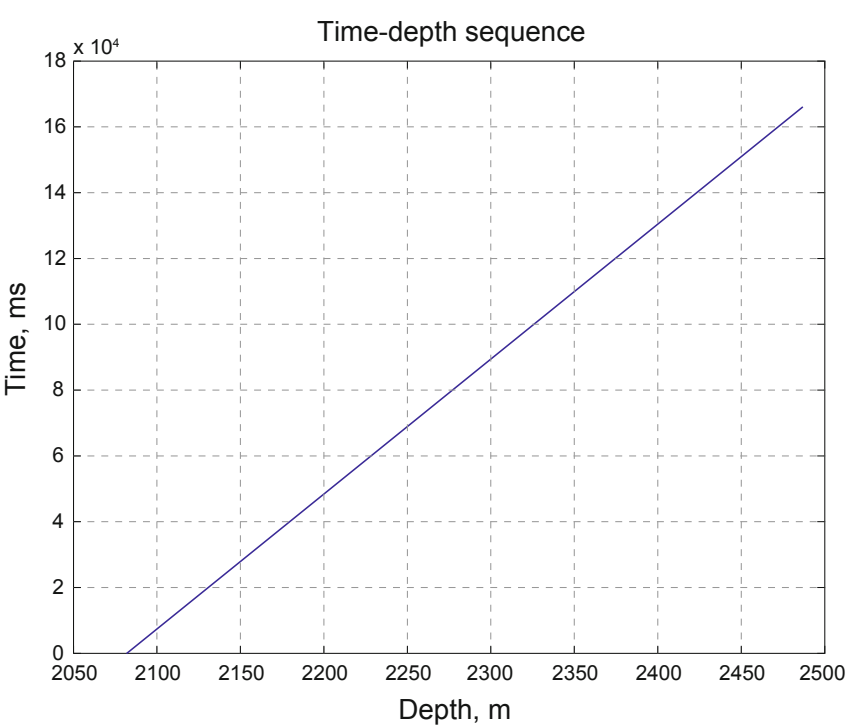

(b) Time-depth sequence

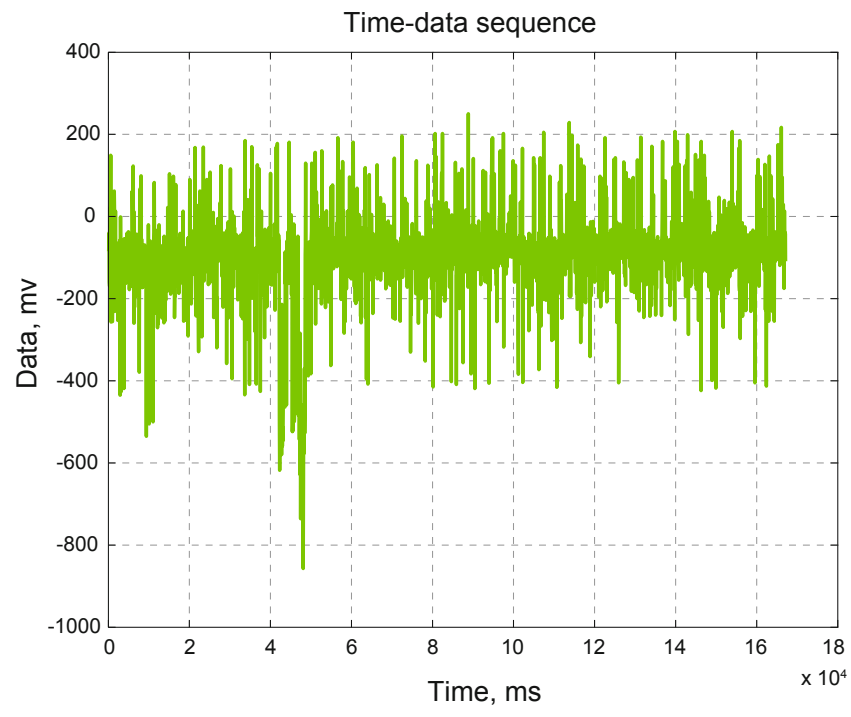

(c) Time-data sequence

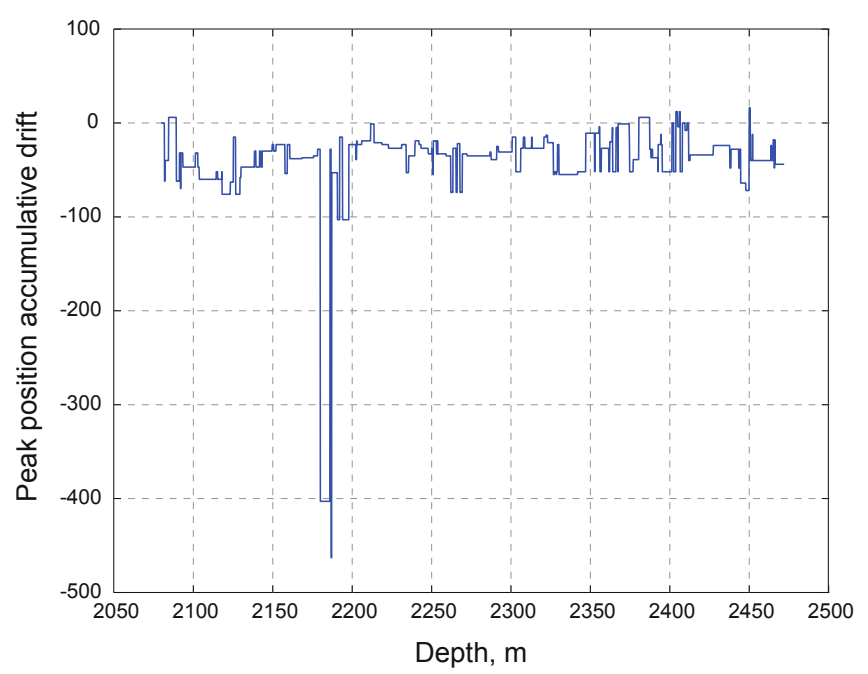

(d) Peak accumulative drift 


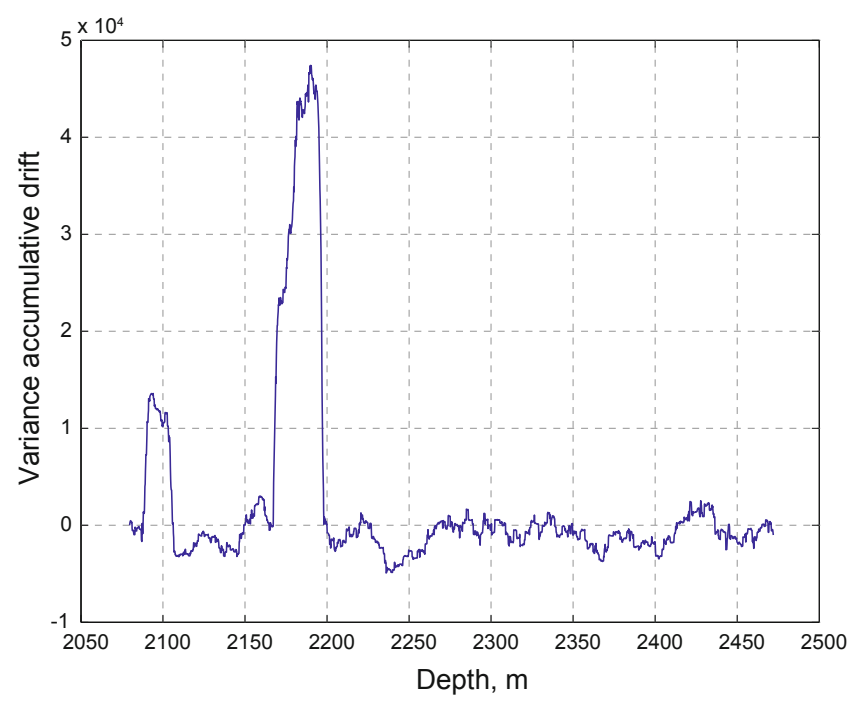

(e) Variance accumulative drift
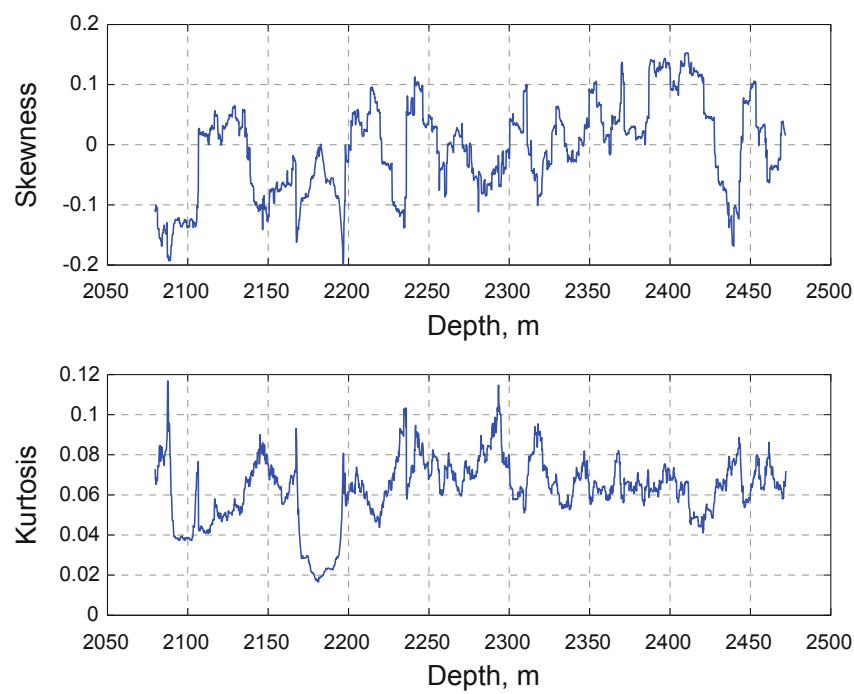

(f) PP-skewness and PP-kurtosis curve

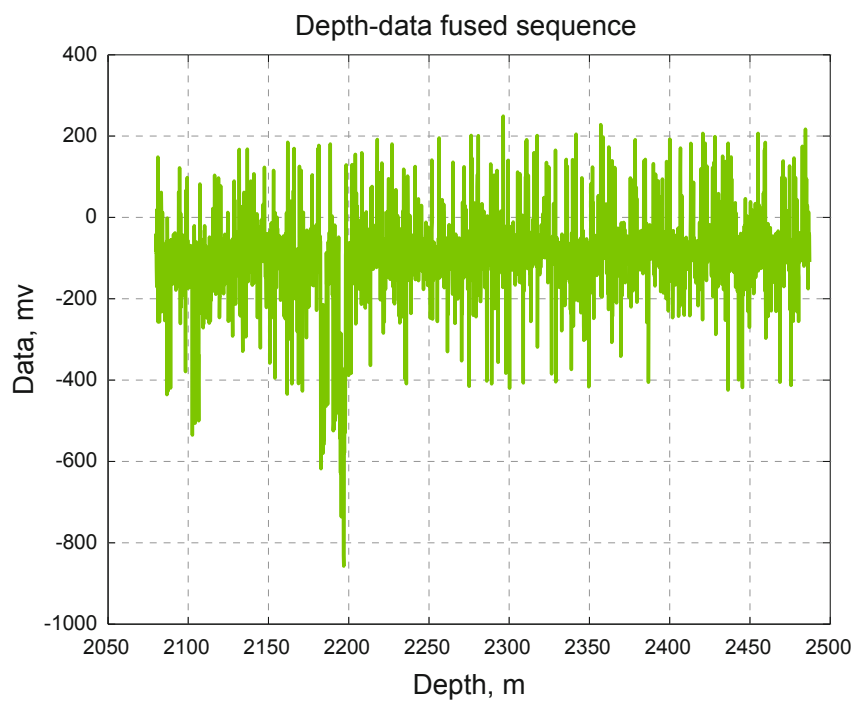

(g) Fused depth-data sequence

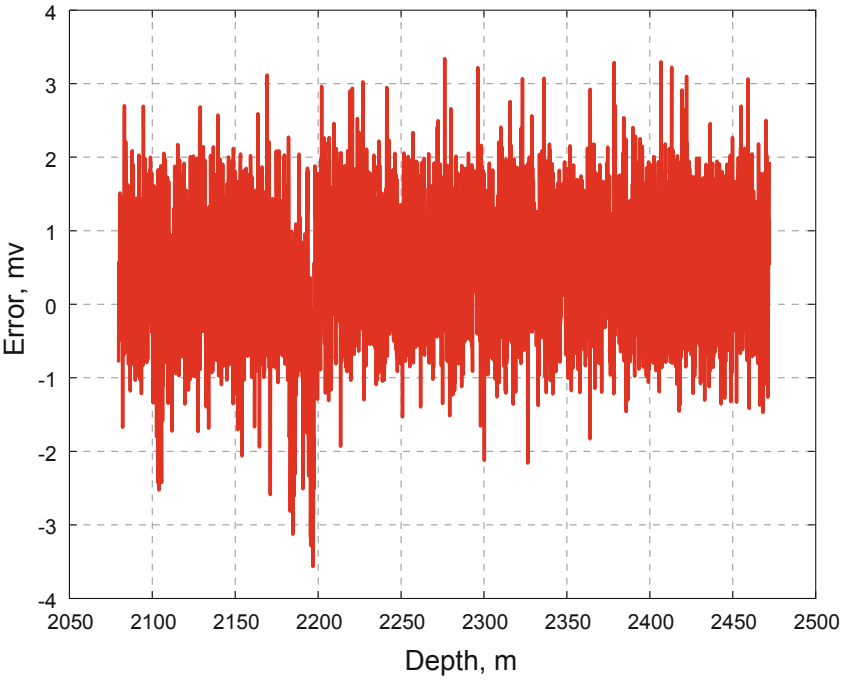

(h) Error curve $<d, \Delta \tilde{r}>$

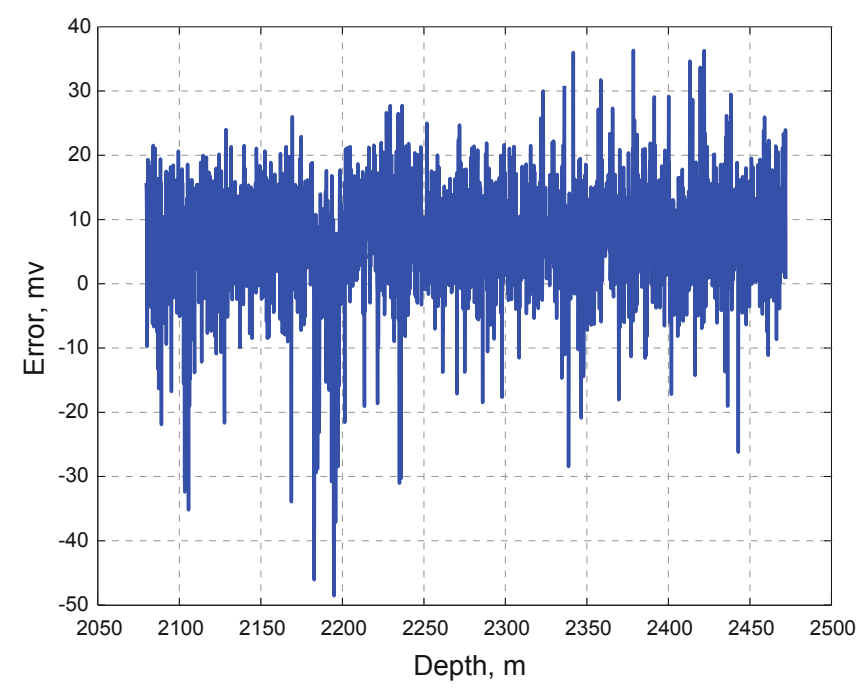

(i) Error curve $<d, \Delta \bar{r}\rangle$

Fig. 4 Time-depth-data fusion results

If the fractal interpolation is not used in each interval, but in the whole depth domain of the acquired data, there will be a long delay. For example, the delay for the sonic curve shown in Fig. 4(a) includes two parts: 1) The first part $T_{1}$ is the time waiting for acquiring all the data of 2675 depth points. If the speed of the cable is $30 \mathrm{~m} / \mathrm{min}, T_{1}$ is (24872079) $/ 30 \times 60=816$ seconds. 2) The second part is the time of interpolation for all sampling points $T_{2}$. On our simulation platform, $T_{2}$ is 240 seconds. However, the time delay of the piecewise fractal interpolation proposed in this paper is approximately 15 seconds on the same platform. Therefore, the piecewise fractal interpolation can improve the real-time processing for the TDTM and logging networks.

\section{Conclusions}

The time-driven transmission method does not need a high-level real-time network. However, the well logging network should keep an accurate synchronization time to 
reduce depth error, which can be achieved with the existing time synchronization solutions. Time synchronization is a main factor for the TDTM to decrease errors. The method to extract fractal intervals based on the change of histogram characteristics is very effective. The piecewise fractal interpolation can obtain high-resolution sequences for data fusion, which meets the demand of highprecision transmission and accurate logging interpretation. Experimental results show that the fused data have less errors and the time-driven transmission method is effective and suitable for the logging networks.

\section{Acknowledgements}

This work was supported by the China National Offshore Oil Corporation under the High Speed Logging Transmission Network based on OFDM and Ethernet Program, and also supported by the UESTC-COSL Joint Laboratory of Electrical Logging. The authors would like to thank Dr. Meng and Mr. Jin for providing the logging data.

\section{References}

Burnside D and Parks T W. Amplitude bound interpolation of sonic well$\log$ data. Acoustics, Speech and Signal Processing. 1990. 4: 19331936 (ICASSP-90)

Chen G L and Cui H J. The testing for normality based on PP-skewness and PP-kurtosis in EV model. Mathematica Applicata. 2004. 17(1): 16-21 (in Chinese)

Hewett T A. Fractal distributions of reservoir heterogeneity and their influence on fluid transport. Society of Petroleum Engineers Annual Technical Conference and Exhibition. New Orleans, USA. 1986

Lian F L, Moyne J R and Tilbury D M. Performance evaluation of control networks: Ethernet, ControlNet, and DeviceNet. IEEE
Control Systems Magazine. 2001. 21(1): 66-83

Li C F. Some remarks on application of fractal analyses in well logging data. Well Logging Technology. 2005. 29(1): 15-20 (in Chinese)

Liu H Q, Peng S M, Zhou Y Y, et al. Discrimination of natural fractures using well logging curve unit. Journal of China University of Geosciences. 2004. 15(4): 372-378

Li Y S and Xiao X C. A study on the fractal characterization of sonic logging signal. Signal Processing. 2002. 18(2): 186-188 (in Chinese)

Lu J A and Li Z B. The self-similarity study of well logging curves. Well Logging Technology. 1996. 6(20): $422-427$ (in Chinese)

Manousopoulos P, Drakopoulos V and Theoharis T. Curve fitting by fractal interpolation. Lecture Notes in Computer Science, Transactions on Computational Science I. Berlin: Springer. 2008. 4750: $85-103$

Mazel D S and Hayes M H. Using iterated function systems to model discrete sequences. IEEE Transactions on Signal Processing. 1992. 40(7): 1724-1734

Meng G S, Zhang L, Zhang C, et al. The principle of the WTS surface communication unit in ECLIPS-5700 logging system. Tuha Oil \& Gas. 2003. 8(4): 354-357 (in Chinese)

Sha Z and Liu Y L. The error of FIF. Applied Mathematics: A Journal of Chinese Universities (Ser. A). 2004. 19(2): 193-202 (in Chinese)

Tang $\mathrm{T}$ Z. Technical present and future developing consideration on EILog logging system. Well Logging Technology. 2007. 31(2): 99102 (in Chinese)

Wang R, Liu Z Y and Zhang Y G. Design and implementation of an oilwell-logging data transport system. Computer Engineering. 2006. 32(4): 236-240 (in Chinese)

$\mathrm{Wu}$ W B, Yao J H and Shen J W. Real-time long range transmission of log data through GPRS. Petroleum Instruments. 2005. 19(4): 72-73 (in Chinese)

Xiao L Z, Xie R H, Chai X Y, et al. Well logging technology for the new century: network-based logging technology. Well Logging Technology. 2003. 27(1): 6-10 (in Chinese)

(Edited by Hao Jie) 